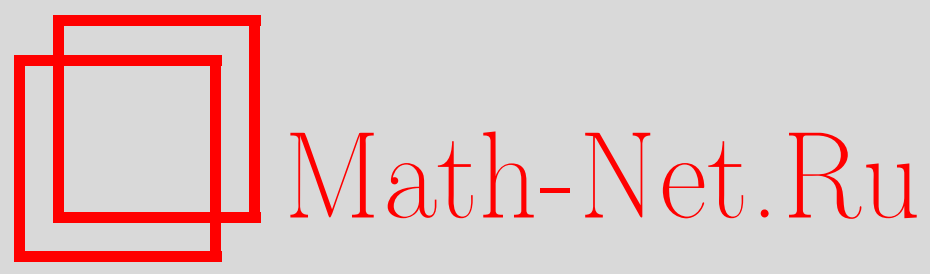

В. М. Тарарин, О с-3-транзитивных группах автоморфизмов циклически упорядоченных множеств, Матем. заметкu, 2002, том 71, выпуск 1, 122-129

DOI: https://doi.org/10.4213/mzm333

Использование Общероссийского математического портала Math-Net.Ru подразумевает, что вы прочитали и согласны с пользовательским соглашением http://www . mathnet.ru/rus/agreement

Параметры загрузки:

IP: 34.227 .88 .159

26 апреля 2023 г., 15:59:52

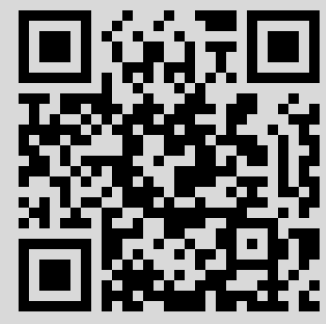




\title{
О С-3-ТРАНЗИТИВНЫХ ГРУППАХ АВТОМОРФИЗМОВ ЦИКЛИЧЕСКИ УПОРЯДОЧЕННЫХ МНОЖЕСТВ
}

\author{
B. М. Тарарин
}

Группа $G$ автоморфизмов циклически упорядоченного множества $\langle X, C\rangle$ называется с-3-транзитивной, если для любых элементов $x_{i}, y_{i} \in X(i=1,2,3), C\left(x_{1}, x_{2}, x_{3}\right)$, $C\left(y_{1}, y_{2}, y_{3}\right)$ найдется элемент $g \in G$ такой, что $g\left(x_{i}\right)=y_{i}, i=1,2,3$. Установлена простота группы всех автоморфизмов цик лически упорядоченного множества в случае ее с-3-транзитивности. Дано описание с-3-транзитивных групп автоморфизмов с абелевым стабилизатором двух точек.

Библиография: 7 названий.

В данной заметке установлена простота групшы всех автоморфизмов циклически упорядоченного множества в случае ее с-3-транзитивности, а такжепоказано, что с-3-транзитивная группа автоморфизмов циклически упорядоченного множества $X, \operatorname{card}(X)$ $\geqslant 4$, с абелевым стабилизатором двух точек изоморфна фактор-групше $\mathbf{P G L} \mathbf{L}_{2}^{+}(P)$ группы $\mathbf{G L}_{2}^{+}(P)$ матриц порядка два с положительными определителями по нормальной подгрупше $P^{*} \mathbf{E}_{2}$ скалярных матрищ для некоторого линейно упорядоченного поля $P$.

Непустое множество $X$ назьвается ииклически упорядоченным множеством (ЦУ множсеством) [1], если на $X$ определено тернарное отношение $C$, обладающее следующими свойствами:

С1) если $C(x, y, z)$, то $x \neq y \neq z \neq x$;

С2) если $x \neq y \neq z \neq x$, то имеет место ровно одно из отношений $C(x, y, z)$ и $C(x, z, y)$

С3) если $C(x, y, z)$, то $C(y, z, x)$;

С4) если $C(x, y, z)$ и $C(x, z, t)$, то $C(x, y, t)$.

Важный пример ЦУ множества - ЦУ множество Т комплексных чисел на единичной окружности, если $C(x, y, z)$ означает, что числа $x, y, z$ следуют друг за другом при обходе против часовой стрелки.

В дальнейшем $C\left(a_{1}, a_{2}, \ldots, a_{n}\right), n \geqslant 3$, означает, что $C\left(a_{i}, a_{j}, a_{k}\right)$ имеет место для всех $i<j<k$. Для элементов $a, b$ ЦУ множества $\langle X, C\rangle$ полагаем $((a, b))=\{x \in X \mid$ $C(a, x, b)\},[[a, b]]=((a, b)) \cup\{a, b\}$. Если $\langle X, C\rangle$ - ЦУ множество и $f-$ подстановка множества $X$, то $f$ называется автоморфизмом ЦУ множества $\langle X, C\rangle$, если из $C(x, y, z)$ следует $C(f(x), f(y), f(z))$ для всех $x, y, z \in X$. Группой автоморфизмов ЦУ множества $\langle X, C\rangle$ назьвается любая подгруппа $G$ группы $A(\langle X, C\rangle)$ всех автоморфизмов Цу 
множества $\langle X, C\rangle$, рассматриваемая как группа, действующая на $X$. Через $е$ обозначаем единичньй элемент группы. Группа $G$ транзитивна, если для любьх $x, y \in X$ найдется $g \in G$ такой, что $g(x)=y$.

Группу $G$ автоморфизмов ЦУ множества $\langle X, C\rangle, \operatorname{card}(X) \geqslant n$, будем назьвать с- $n$ транзитивной $(n \geqslant 3)$, если для любых элементов $x_{i}, y_{i} \in X, i=1, \ldots, n, C\left(x_{1}, \ldots, x_{n}\right)$, $C\left(y_{1}, \ldots, y_{n}\right)$ найдется элемент $g \in G$ такой, что $g\left(x_{i}\right)=y_{i}, i=1, \ldots, n$.

ПРИмЕР. Простейшим примером с-3-транзитивной группы является циклическая группа третьего порядка $A(\langle X, C\rangle)$ автоморфизмов ЦУ множества $\langle X, C\rangle$, состоящего из трех элементов $x_{1}, x_{2}, x_{3}$ с отношением $C=\left\{\left(x_{1}, x_{2}, x_{3}\right),\left(x_{2}, x_{3}, x_{1}\right),\left(x_{3}, x_{1}, x_{2}\right)\right\}$.

Пусть $G$ - группа автоморфизмов ЦУ множества $\langle X, C\rangle$ и $a \in X$. Тогда из условий $\mathrm{C} 1)-\mathrm{C} 4)$ следует, что отношение $\leqslant a$ на $X$, определяемое по правилу:

C5) $x \leqslant a$, если и только если $x=a$ или $x=y$, или $C(a, x, y)$ является отношением линейного порядка, причем, для любого элемента $g$, принадлежащего стабилизатору $G_{a}=\{g \in G \mid g(a)=a\}$ в группе $G$ точки $a$, вьполняется свойство: из $x \leqslant a$ следует $g(x) \leqslant a g(y)$ для любых $x, y \in X$. Таким образом, справедлива

Лемма 1. Стабилизатор $G_{a}$ в группе $G$ автоморфизмов ЦУУ мнохсества $\langle X, C\rangle$ любой точки $a \in X$ является группой автоморфизмов линейно упорядоченного множсества $\langle X, \leqslant a\rangle$.

Заметим, что групша $G_{a}$ действует точно на множестве $X \backslash\{a\}$ и поэтому можно считать, что $G_{a}$ является группой автоморфизмов линейно упорядоченного множества (ЛУ множества) $\langle X \backslash\{a\}, \leqslant a\rangle$.

Необходимые сведения по теории групп автоморфизмов ЛУ множеств содержатся в [2].

Непосредственно из определения с- $n$-транзитивной группы автоморфизмов вытекает

Лемма 2. 1) С-n-транзитивная группа автоморфизмов является транзитивной.

2) С-n-транзитивная группа автоморфизмов является c-t-транзитивной для любого натурального числа $t, 3 \leqslant t \leqslant n$.

Группа $G \neq\{e\}$ автоморфизмов ЛУ множества $\langle X, \leqslant\rangle$ назьвается о- $n$-транзитивной $(n \geqslant 2)$ [2], если для любых элементов $x_{1}<\cdots<x_{n}$ и $y_{1}<\cdots<y_{n}$ множества $X$ найдется $g \in G$ такой, что $g\left(x_{i}\right)=y_{i}, i=1, \ldots, n$. Очевидно, что о- $n$-транзитивная группа транзитивна.

Лемма 3. Группа $G$ автоморфизмов ЦУУ множества $\langle X, C\rangle, \operatorname{card}(X) \geqslant 4$, является с-п-транзитивной тогда и только тогда, когда стабилизатор $G_{a}$ в группе $G$ любой точки $a \in X$ является о- $(n-1)$-транзитивной группой автоморфизмов ЛУ множества $\langle X \backslash\{a\}, \leqslant a\rangle$.

ДокАЗАТЕЛЬСТво. Если $G$ - c- $n$-транзитивная группа автоморфизмов ЦУ множества $\langle X, \mathrm{C}\rangle, \operatorname{card}(X) \geqslant 4$, и $a \in X$, то $\operatorname{card}(X \backslash\{a\}) \geqslant 3$ и, следовательно, найдутся элементы $a_{1}, a_{2}, a_{3} \in X \backslash\{a\}$ такие, что $C\left(a, a_{1}, a_{2}, a_{3}\right)$. Из последнего отношения имеем $C\left(a, a_{1}, a_{2}\right), C\left(a, a_{2}, a_{3}\right)$, откуда в силу леммы 2 найдется $g \in G$ такой, что $g(a)=a$, $g\left(a_{1}\right)=a_{2}, g\left(a_{2}\right)=a_{3}$. Таким образом, $g \in G_{a}$ и $g \neq e$ и, следовательно, в силу леммы 1 
$G_{a}$ является неединичной группой автоморфизмов ЛУ множества $\langle X \backslash\{a\}, \leqslant a\rangle$, откуда, в частности, следует, что $X \backslash\{a\}$ - бесконечное множество. Если $x_{1}<a \cdots<a x_{n-1}$ и $y_{1}<_{a} \cdots<_{a} y_{n-1}-$ произвольные элементы ЛУ множества $\langle X \backslash\{a\}$, $\leqslant a\rangle$, то по определению $\mathrm{C} 5)$ порядка $\leqslant a$ получаем $C\left(a, x_{1}, \ldots, x_{n-1}\right), C\left(a, y_{1}, \ldots, y_{n-1}\right)$ и в силу с- $n$-транзитивности группы $G$ найдется $g \in G$ такой, что $g(a)=a, g\left(x_{i}\right)=y_{i}$, $i=1, \ldots, n-1$. Следовательно, стабилизатор $G_{a}$ является о- $(n-1)$-транзитивной группой автоморфизмов ЛУ множества $\langle X \backslash\{a\}, \leqslant a\rangle$.

Обратно, если стабилизатор $G_{a}$ в групе $G$ автоморфизмов ЦУ множества $\langle X, C\rangle$ любой точки $a \in X$ является о- $(n-1)$-транзитивной $(n \geqslant 3)$ группой автоморфизмов ЛУ множества $\langle X \backslash\{a\}, \leqslant a\rangle$, то легко заметить, что множество $X \backslash\{a\}$ бесконечно и группа $G$ действует транзитивно на $X$. Пусть $C\left(x_{1}, \ldots, x_{n}\right), C\left(y_{1}, \ldots, y_{n}\right), x_{i}, y_{i} \in X$. Найдется элемент $g \in G$ такой, что $g\left(x_{1}\right)=y_{1}$. Тогда имеем $C\left(y_{1}, g\left(x_{2}\right), \ldots, g\left(x_{n}\right)\right)$. По определению С 5$)$ порядка $\leqslant a$ получаем $g\left(x_{2}\right)<_{y_{1}} \cdots<_{y_{1}} g\left(x_{n}\right)$ и $y_{2}<_{y_{1}} \cdots<_{y_{1}} y_{n}$, откуда по предположению $h\left(g\left(x_{i}\right)\right)=y_{i}, i=2, \ldots, n$, для некоторого элемента $h \in G_{y_{1}}$. Следовательно, $h g\left(x_{i}\right)=y_{i}, i=1, \ldots, n$, и поэтому $G$ является с- $n$-транзитивной группой автоморфизмов. Лемма 3 доказана.

Пусть $G$ - с-3-транзитивная группа автоморфизмов ЦУ множества $\langle X, C\rangle, \operatorname{card}(X)$ 7 4. Тогда непосредственно из леммы 3 следует, что ЛУ множество $\langle X \backslash\{a\}, \leqslant a\rangle, a \in X$, не обладает ни наибольшим, ни наименьшим элементом, откуда в силу определения C5) порядка $\leqslant a$ получаем, что $((a, b)) \neq \oslash$ для $b \in X, b \neq a$. Следовательно, имеет место

Лемма 4. Пусть $G$ - с-3-транзитивная группа автоморфизмов ЦУУ множества $\langle X, C\rangle, \operatorname{card}(X) \geqslant 4$. Тогда $((a, b)) \neq \oslash$ для любых әлементов $a, b \in X, a \neq b$.

Несложные рассуждения показывают, что справедлива

Лемма 5. Орраничение стабилизатора $G_{a}$ в әруппе $G=A(\langle X, C\rangle)$ всех автоморфизмов ЦУ множества $\langle X, C\rangle$ любой точки $а \in X$ на множество $X \backslash\{a\}$ совпадает $c$ әруппой $A(\langle X \backslash\{a\}, \leqslant a\rangle)$ всех автоморфизмов ЛУ множества $\langle X \backslash\{a\}, \leqslant a\rangle$.

Лемма 6. С-3-транзитивная группа $A(\langle X, C\rangle)$ всех автоморфизмов ЦУ множества $\langle X, C\rangle, \operatorname{card}(X) \geqslant 4$, является c- - -транзитивной группой для любого натурального числа $n \geqslant 3$.

ДокАЗАТЕЛЬСТво. Пусть вьполнено условие леммы и $G=A(\langle X, C\rangle)$. Тогда в силу лемм 3 и 5 для любой точки $a \in X$ стабилизатор $G_{a}$ является о-2-транзитивной групшой $A(\langle X \backslash\{a\}, \leqslant a\rangle)$ всех автоморфизмов ЛУ множества $\langle X \backslash\{a\}, \leqslant a\rangle$ и, следовательно, о-2-транзитивной решеточно упорядоченной группой автоморфизмов, откуда в силу [2, c. 69] получаем, что $G_{a}-$ о- $(n-1)$-транзитивная группа автоморфизмов ЛУ множества $\langle X \backslash\{a\}, \leqslant a\rangle$ для любого натурального числа $n \geqslant 3$. Поэтому по лемме 3 группа $G$ является с- $n$-транзитивной для любого $n \geqslant 3$. Лемма 6 доказана.

ТЕорема 1. Если группа $A(\langle X, C\rangle)$ всех автоморфизмов ииклически упорядоченного мнохества $\langle X, C\rangle$ является с-3-транзитивной, то она простая.

ДокАЗАТЕЛЬСТво. Пусть грушпа $G=A(\langle X, C\rangle)$ является с-3-транзитивной. Если $\operatorname{card}(X)=3$, то $G$ - циклическая группа третьего порядка из примера и, следовательно, простая. В дальнейшем предполагаем, что $\operatorname{card}(X) \geqslant 4$. 
Автоморфизм $f \in G$ ЦУ множества $\langle X, C\rangle$ будем назьвать автоморфизмом с ограниченным носителем $\operatorname{supp} f=\{x \in X \mid f(x) \neq x\}$, если существуют $t_{1}, t_{2} \in X$, $t_{1} \neq t_{2}$, такие, что $f(x)=x$ для всех $x \in\left[\left[t_{1}, t_{2}\right]\right]$. Покажем, что грушпа $G$ порождается автоморфизмами с ограниченньми носителями. Пусть $g \in G$. Если $g=e$, то $g$ имеет ограниченный носитель. Предположим, что $g \neq e$ и $g(a)=a$ для некоторой точки $a \in X$. Тогда в силу леммы $1 g$ является автоморфизмом ЛУ множества $\langle X, \leqslant a\rangle$. Пусть для определенности $a_{1}<_{a} g\left(a_{1}\right)=a_{0}$ для некоторой точки $a_{1} \in X \backslash\{a\}$. Тогда по определению порядка $\leqslant a$ и лемме 4 найдется точка $b \in X \backslash\{a\}, b<_{a} a_{1}$, и последовательность $\left\{a_{n} \mid n=2,3, \ldots\right\}$ такие, что $a_{0}>_{a} a_{1}>_{a} a_{2}>_{a} \cdots>_{a} b$. Пусть $c-$ точная нижняя грань последовательности $\left\{a_{n}\right\}$ в пополнении $\overline{(X \backslash\{a\})}$ ЛУ множества $\langle X \backslash\{a\}, \leqslant a\rangle$. По условию теоремы и лемме 3 найдутся автоморфизмы $g_{n} \in G_{a}$, $n=1,2, \ldots$, такие, что $g_{n}\left(a_{n+1}\right)=a_{n}, g_{n}\left(a_{n}\right)=a_{n-1}$. Положим для $x \in X$

$$
f(x)= \begin{cases}x, & \text { если } x \leqslant a c, \\ g_{n}(x), & \text { если } a_{n+1} \leqslant_{a} x \leqslant a \\ g(x), & \text { если } a_{1} \leqslant_{a} x .\end{cases}
$$

Несложно заметить, что $f$ - автоморфизм ЛУ множества $\left\langle X, \leqslant_{a}\right\rangle$ и, следовательно, в силу леммы $5 f \in G$, причем, очевидно, $f$ и $s=f^{-1} g$ имеют ограниченные носители, откуда получаем, что $g$ является произведением автоморфизмов $f$ и $s$ с ограниченньми носителями. Пусть теперь $g \neq e$-произвольньй элемент групшы $G$. Тогда $g(x) \neq x$ для некоторой точки $x \in X$. Пусть $y \in X, y \neq x, y \neq g(x)$. По условию теоремы и лемме 3 найдется $f \in G$ такой, что $f(y)=y$ и $f(x)=g(x)$, откуда $g=f s, s(x)=x$, где $s=f^{-1} g$. По доказанному вьше $f$ и $s$ являются произведениями автоморфизмов с ограниченньми носителями, поэтому $g$ представим в виде произведения автоморфизмов с ограниченньпи носителями.

Пусть $H$ - нормальная подгрупша группы $G, H \neq\{e\}$. Предположим сначала, что $H_{1}=H \cap G_{a} \neq\{e\}$ для некоторой точки $a \in X$. Тогда, очевидно, $H_{1}$ действует точно на множестве $X \backslash\{a\}$ и в силу лемм $1,3,5$ является неединичной нормальной подгруппой о-2-транзитивной группы $G_{a}$ всех автоморфизмов ЛУ множества $\langle X \backslash\{a\}, \leqslant a\rangle$, следовательно ([3] или [2, с. 87]), содержит группу $B$ всех автоморфизмов $h$ ЛУ множества $\left\langle X \backslash\{a\}, \leqslant_{a}\right\rangle$, носитель $\operatorname{supp} h=\{x \in X \backslash\{a\} \mid h(x) \neq x\}$ которых ограничен сверху и снизу в ЛУ множестве $\langle X \backslash\{a\}, \leqslant a\rangle$. Пусть произвольньй автоморфизм $f \in G$ имеет ограниченньй носитель на ЦУ множестве $\langle X, C\rangle$, например, $f(x)=x$ для всех $x \in\left[\left[t_{1}, t_{2}\right]\right], t_{1} \neq t_{2}$. В силу леммы 4 найдется точка $b \in\left(\left(t_{1}, t_{2}\right)\right)$ и в силу транзитивности группы $G$ найдется $s \in G$ такой, что $s(b)=a$. Тогда $f_{1}=s f s^{-1} \in G_{a}$ и $f_{1}(x)=x$ для всех $x \in\left[\left[a, s\left(t_{2}\right)\right]\right] \cup\left[\left[s\left(t_{1}\right), a\right]\right]$ и, следовательно, $f_{1}(x)=x$ для $x \leqslant a s\left(t_{2}\right)$ и $x \geqslant_{a} s\left(t_{1}\right)$. Таким образом, $f_{1}$ является ограниченным автоморфизмом ЛУ множества $\langle X \backslash\{a\}, \leqslant a\rangle$ и, следовательно, $f_{1} \in B \subseteq H_{1}$, откуда вытекает, что $f=s^{-1} f_{1} s \in H$. Поэтому группа $H$ содержит все ограниченные автоморфизмы ЦУ множества $\langle X, C\rangle$ и по доказанному вьше $H=G$.

Теперь предположим, что нормальная подгруппа $H \neq\{e\}$ группы $G$ удовлетворяет условию $H \cap G_{t}=\{e\}$ для всех $t \in X$. Пусть $p \in H, p \neq e$ и $a \in X$. По предположению $p(a) \neq a$. В силу леммы 4 найдутся $b, c \in X$ такие, что $C(a, b, c, p(a))$ и $C\left(a, b, c, p^{-1}(a)\right)$. Из последнего отношения следует $C(p(a), p(b), p(c), a)$ и, следовательно, по С3) имеем $C(a, p(a), p(b), p(c))$, откуда по C4) ввиду $C(a, b, c, p(a))$ получаем 
$C(a, b, c, p(a), p(b), p(c))$. В силу леммы 4 найдется $d \in X$ такой, что $C(a, b, c, p(a), p(b)$, $p(c), d)$, откуда имеем $C(a, b, p(a), p(b))$ и $C(a, c, p(a), d)$. По условию теоремы и лемме 6 найдется элемент $s \in G$ такой, что $s(a)=a, s(b)=c, s(p(a))=p(a), s(p(b))=d$. Заметим, что $(s p)(b)=s(p(b))=d \neq p(c)=p(s(b))=(p s)(b)$ и $s(a)=a, s(p(a))=p(a)$. Из последних равенств вытекает равенство $\left(s p s^{-1}\right)(a)=p(a)$, откуда в силу предположения о нормальной подгруппе $H$ получаем $s p s^{-1}=p$ и, следовательно, $s p=p s$, что противоречит полученному выше неравенству. Таким образом, групша $G$ не обладает нормальной подгруппой $H \neq\{e\}$, удовлетворяющей условию $H \cap G_{t}=\{e\}$ для всех $t \in X$.

Из изложенного вытекает, что $G$ - простая группа. Теорема 1 доказана.

ЛЕмма 7. Если G-с-3-транзитивная группа автоморфизмов ЦУ множества $\langle X, C\rangle$ с абелевым стабилизатором точки, то $\operatorname{card}(X)=3 u G-$ ииклическая группа третьего порядка.

ДокАЗАТЕЛЬСтво. Пусть группа $G$ удовлетворяет условию леммы. Если $\operatorname{card}(X)$ $=3$, то $G$ - циклическая группа третьего порядка из примера. $\operatorname{Ecли~} \operatorname{card}(X) \geqslant 4$, то в силу леммы 3 стабилизатор $G_{a}, a \in X$, является о-2-транзитивной групой автоморфизмов ЛУ множества $\langle X \backslash\{a\}, \leqslant a\rangle$ и поэтому не может быть абелевой групой. Лемма 7 доказана.

ТЕОРема 2. Пусть $G$ - с-3-транзитивная группа автоморфизмов чиклически упорядоченного множества $\langle X, C\rangle, \operatorname{card}(X) \geqslant 4$, с абелевым стабилизатором двух точек. Тогда группа $G$ изоморфна группе

$$
F^{+}=\left\{\frac{a x+b}{c x+d} \mid a, b, c, d \in P, \quad a d-b c>0\right\}
$$

относительно суперпозичии дробно-линейных функиий с коэффичиентами из некоторого линейно упорядоченного поля $P$.

ДокАЗАТЕЛЬСТВо. Пусть $G$ - с-3-транзитивная группа автоморфизмов ЦУ множества $\langle X, C\rangle, \operatorname{card}(X) \geqslant 4$, с абелевым стабилизатором $A$ двух точек $a_{1}, a_{2} \in X$. Ввиду леммы 7 точки $a_{1}, a_{2}$ различны. Положим $\infty=a_{1}$ и $0=a_{2}$. По условию теоремы в силу леммы 3 стабилизатор $G_{\infty}$ в группе $G$ точки $\infty$ действует точно и о-2-транзитивно на ЛУ множестве $X^{\prime}=\langle X \backslash\{\infty\}, \leqslant a\rangle$, причем стабилизатор $A=\left\{g \in G_{\infty} \mid g(0)=0\right\}$ в группе $G_{\infty}$ точки 0 является абелевым. Следовательно [4], на ЛУ множестве $X^{\prime}$ можно определить структуру линейно упорядоченного поля $\langle P,+, \cdot, 0,1\rangle$ таким образом, что ограничение групшы $G_{\infty}$ на множество $X^{\prime}$ совпадает с группй $H=\{\varphi(a, b) \mid a, b, \in P$, $a>_{\infty} 0, \varphi(a, b)(x)=a x+b$ для $\left.x \in P\right\}$. Доопределив элементы групшы $H$ на все множество $X$, полагая $\varphi(a, b)(\infty)=\infty$, можно считать, что $G_{\infty}=H$ и $A=\{\varphi(a, 0) \mid a \in P$, $\left.a>_{\infty} 0\right\}$. Положим $X^{\prime}=P, X=P \cup\{\infty\}$. В дальнейшем $\leqslant \infty$ обозначаем просто $\leqslant$, a также полагаем $\varphi(a)=\varphi(a, 0)$.

По изложенному вьше и определению $C 5)$ порядка $\leqslant$ имеем $C(\infty,-1,0,1)$, в частности, $C(0,1, \infty)$ и $C(\infty,-1,0)$. По условию теоремы найдется элемент $\lambda \in G$ такой, что $\lambda(0)=\infty, \lambda(1)=-1, \lambda(\infty)=0$. Заметим, что если $\lambda(a)=-a, a \in P, a>0$, то $a=1$. Действительно, пусть для определенности $a>1$. Тогда имеем $C(0,1, a)$ и $C(\infty,-a,-1)$, 
откуда из первого отношения получаем $C(\lambda(0), \lambda(1), \lambda(a))$ и $C(\infty,-1,-a)$, что противоречит второму отношению. Следовательно, $a=1$.

Так как $\lambda^{2}(0)=0, \lambda^{2}(\infty)=\infty$, то $\lambda^{2} \in A$ и, следовательно, $\lambda^{2}=\varphi(a)$ для некоторого элемента $a \in P, a>0$. Так как из равенств

$$
\lambda^{2}(1)=\varphi(a)(1)=a, \quad \lambda^{2}(-1)=\varphi(a)(-1)=-a
$$

ввиду $\lambda(1)=-1$ вытекает равенство $\lambda(a)=-a$, то в силу замечания $a=1$ и $\lambda^{2}=$ $\varphi(1)(1)=e$. Имеем $\lambda \varphi(a) \lambda(\infty)=\infty, \lambda \varphi(a) \lambda(0)=0$ для любого элемента $\varphi(a) \in A$, откуда получаем, что $\lambda \varphi(a) \lambda \in A$ для всех $\varphi(a) \in A$ и, следовательно, ввиду $\lambda^{2}=e$ отображение $\gamma: A \rightarrow A, \gamma(\varphi(a))=\lambda \varphi(a) \lambda$, является автоморфизмом групшы $A$, причем, очевидно, $\gamma^{2}$ - тождественный автоморфизм. В силу замечания $\lambda(a) \neq-a$ для всех $a \in P, a>0, a \neq 1$, откуда ввиду равенств $\lambda(1)=-1, \lambda^{2}=e$ получаем $\lambda \varphi(a) \lambda(-1) \neq \varphi(a)(-1)$ и, значит, $\gamma(\varphi(a)) \neq \varphi(a)$ для всех $a \in P, a>0, a \neq 1$, т.е. для всех $\varphi(a) \in A, \varphi(a) \neq e$. Так как $\gamma$ - автоморфизм порядка два (т.е. инволюция) абелевой группы $A$ без кручения, не имеющий неподвижных точек, отличных от единичного элемента групшы $A$, то так же, как в $[5, \S 116$, п. ж, с. 317$]$, показьвается, что $\gamma(\varphi(a))=(\varphi(a))^{-1}=\varphi\left(a^{-1}\right)$ для всех $\varphi(a) \in A$. Для любого элемента $a \in P, a>0, \mathrm{~B}$ силу равенства $\gamma(\varphi(a))=\lambda \varphi(a) \lambda=\varphi\left(a^{-1}\right)$ имеем $\lambda \varphi(a)=\varphi\left(a^{-1}\right) \lambda$, откуда получаем

$$
\lambda(a)=\lambda \varphi(a)(1)=\varphi\left(a^{-1}\right) \lambda(1)=\varphi\left(a^{-1}\right)(-1)=-a^{-1}
$$

и

$$
\lambda(-a)=\lambda \varphi(a)(-1)=\varphi\left(a^{-1}\right) \lambda(-1)=\varphi\left(a^{-1}\right)(1)=a^{-1} .
$$

Следовательно, $\lambda(x)=-x^{-1}$ для всех $x \in P, x \neq 0$ и $\lambda(0)=\infty, \lambda(\infty)=0$.

Пусть элемент $g \in G$. Если $g(\infty)=\infty$, то $g \in G_{\infty}$. Если $g(\infty) \neq \infty$, то в силу того, что группа $G_{\infty}$ действует транзитивно на множестве $P$, найдется $s \in G_{\infty}$ такой, что $g(\infty)=s(0)=s \lambda(\infty)$ и, следовательно, $\lambda^{-1} s^{-1} g=r \in G_{\infty}$ и $g=s \lambda r$ для некоторых $s, r \in G_{\infty}$. Таким образом, $G=G_{\infty} \cup\left\{s \lambda r \mid s, r \in G_{\infty}\right\}$, в частности, группа $G$ порождается элементами стабилизатора $G_{\infty}$ и элементом $\lambda$.

На множестве $X=P \cup\{\infty\}$ действует точно групша

$$
F^{+}=\left\{\frac{a x+b}{c x+d} \mid a, b, c, d \in P, \quad a d-b c>0\right\} .
$$

Покажем, что $G=F^{+}$. Пусть $g \in G$. Если $g(\infty)=\infty$, то $g \in G_{\infty}$ и $g(x)=\varphi(a, b)(x)=$ $a x+b, a>0$, откуда, очевидно, следует, что $g \in F^{+}$. Если $g(\infty) \neq \infty$, то по доказанному выше $g=\varphi\left(a_{1}, b_{1}\right) \lambda \varphi(c, d)$ для некоторых $\varphi\left(a_{1}, b_{1}\right), \varphi(c, d) \in G_{\infty}$ и, следовательно, $g(x)=\left(\left(b_{1} c\right) x+\left(b_{1} d-a_{1}\right)\right) /(c x+d)$. Так как $a_{1}>0, c>0$, то элементы $a=b_{1} c$, $b=b_{1} d-a_{1}, c$ и $d$ удовлетворяют соотношению $a d-b c=a_{1} c>0$ и поэтому $g \in F^{+}$. Таким образом, $G \subseteq F^{+}$. Обратно, пусть $\omega(x)=(a x+b) /(c x+d) \in F^{+}$. Если $c=0$, то $\omega(x)=a x / d+b / d$ и $a / d>0$, откуда получаем $\omega \in G$. Если $c \neq 0$, то непосредственная проверка показывает, что

$$
\omega=\varphi\left(\frac{a d-b c}{c^{2}}, \frac{a}{c}\right) \lambda \varphi\left(1, \frac{d}{c}\right)
$$


и, следовательно, ввиду $a d-b c>0$ получаем, что $\omega \in G$. Таким образом, $F^{+} \subseteq G$. Из изложенного вытекает равенство $G=F^{+}$. Теорема 2 доказана.

Пусть $P$ - линейно упорядоченное поле, $P^{*}=P \backslash\{0\}$. Пусть $\mathbf{G L}_{2}^{+}(P)-$ група матрицпорядка два над полем $P$ с положительньми определителями, $P^{*} \mathbf{E}_{2}-$ подгруппа скалярных матриц группы $\mathbf{G L}_{2}^{+}(P), \mathbf{P G L}_{2}^{+}(P)$ - фактор-группа групшы $\mathbf{G L}_{2}^{+}(P)$ по нормальной подгрупше $P^{*} \mathbf{E}_{2}$.

ТЕорема 3. Всякая с-3-транзитивная группа $G$ автоморфизмов ичклически упорядоченного множества $\langle X, C\rangle, \operatorname{card}(X) \geqslant 4$, с абелевым стабилизатором двух точек изоморфна группе $\mathbf{P G L}_{2}^{+}(P)$ над некоторым линейно упорядоченным полем $P$. Обратно, для всякого линейно упорядоченного поля $P$ группа $\mathbf{P G L}_{2}^{+}(P)$ изоморфна с-3-транзитивной группе автоморфизмов с абелевым стабилизатором двух точек некоторого ииклически упорядоченного мнохества $\langle X, C\rangle$.

ДокАЗАТЕЛЬСТво. Если $G$ - с-3-транзитивная группа автоморфизмов ЦУ множества $\langle X, C\rangle, \operatorname{card}(X) \geqslant 4$, с абелевым стабилизатором двух точек, то в силу теоремы 2 $G$ изоморфна группе $F^{+}$над некоторым линейно упорядоченным полем $P$. Непосредственная проверка показывает, что соответствие, сопоставляющее элементу

$$
\omega(x)=\frac{a x+b}{c x+d} \in F^{+}
$$

смежный класс $\left(\begin{array}{ll}a & b \\ c & d\end{array}\right) \cdot P^{*} \mathbf{E}_{2}$ групшы $\mathbf{G L}_{2}^{+}(P)$ по подгрупше $P^{*} \mathbf{E}_{2}$, является изоморфизмом групп $F^{+}$и $\mathbf{P G L}_{2}^{+}(P)$. Следовательно, группа $G$ изоморфна группе $\mathbf{P G L}_{2}^{+}(P)$.

Обратно, пусть $P$ - линейно упорядоченное поле. Положим $X=P \cup\{\infty\}$. На множестве $X$ определим циклический порядок, полагая:

$$
\begin{aligned}
& C(x, y, z) \text {, если } x, y, z \in P \text { и } x<y<z \text { или } z<x<y, \text { или } y<z<x ; \\
& C(\infty, x, y) \text {, если } x, y \in P \text { и } x<y ; \\
& C(x, \infty, y) \text {, если } x, y \in P \text { и } y<x ; \\
& C(x, y, \infty) \text {, если } x, y, \in P \text { и } x<y .
\end{aligned}
$$

По доказанному выше група $\mathbf{P G L}_{2}^{+}(P)$ изоморфна групе $F^{+}$, действующей точно на множестве $X$. Непосредственная проверка с использованием леммы 3 показывает, что $F^{+}-$c-3-транзитивная группа автоморфизмов Цу множества $\langle X, C\rangle$ с абелевым стабилизатором двух точек. Теорема 3 доказана.

СЛЕДСТВИЕ. С-3-транзитивная әруппа $G$ автоморфизмов с единичным стабилизатором трех точек ииклически упорядоченного множсества $\mathbf{T}$ изоморфна проективной специальной линейной группе $\mathbf{P S L}_{2}(\mathbb{R})$ над полем $\mathbb{R}$ вещественных чисел.

ДокАЗАТЕЛЬСТво. Пусть $G$ с-3-транзитивная группа автоморфизмов ЦУ множества $\mathbf{T}$ и стабилизатор трех точек $a_{1}, a_{2}, a_{3} \in \mathbf{T}$ является единичной группой. Тогда в силу с-3-транзитивности группы $G$ и леммы 3 точки $a_{1}, a_{2}, a_{3}$ различны и стабилизатор любых трех различных точек из Т единичен. Зафиксируем элементы $\infty$ и 0 , $\infty \neq 0$, множества $\mathbf{T}$. В силу леммы 3 стабилизатор $G_{\infty}$ точки $\infty$ действует точно и о-2-транзитивно на ЛУ множестве $X^{\prime}=\langle X \backslash\{\infty\}, \leqslant \infty\rangle$, откуда, как несложно заметить, следует, что стабилизатор $A$ в группе $G_{\infty}$ точки 0 действует транзитивно на множестве 
$X^{\prime \prime}=((0, \infty))$. Так как по условию следствия стабилизатор трех различных точек в группе $G$ единичен, то $g(x) \neq x$ для любых $x \in X^{\prime \prime}, g \in A, g \neq e$, и, следовательно, $A$ является регулярной группой порядковых автоморфизмов ЛУ множества $\left\langle X^{\prime \prime}, \leqslant \infty\right\rangle$, порядково изоморфного ЛУ множеству $\mathbb{R}$ вешественных чисел. Несложные рассуждения показывают, что группа $A$ относительно порядка $\leqslant$ (определенного по правилу $g \leqslant h$, $g, h \in A$, тогда и только тогда, когда $g(x) \leqslant h(x)$ для всех $x \in X^{\prime \prime}$ ) является архимедовой линейно упорядоченной группой и, следовательно [6], абелевой. Таким образом, $G-$ c-3-транзитивная группа автоморфизмов с абелевым стабилизатором $A$ двух точек $\infty$ и 0 , откуда по теореме 3 следует, что группа $G$ изоморфна группе $\mathbf{P G L}_{2}^{+}(P)$ для некоторого линейно упорядоченного поля $P$, причем в силу доказательств теорем 2,3 поле $P$ определено на ЛУ множестве $X^{\prime}$, порядково изоморфном ЛУ множеству вешественных чисел, и, следовательно, ввиду [6. с. 153] и [1. с. 190] $P$ изоморфно полю $\mathbb{R}$ вещественных чисел. Так как, очевидно, $\mathbf{P G L}_{2}^{+}(\mathbb{R})$ совпадает с $\mathbf{P S L}_{2}(\mathbb{R})$, то следствие доказано.

Заметим, что в силу теоремы Жордана-Диксона [7. с. 119] для всякого линейно упорядоченного поля $P$ группа $\mathbf{P S L}_{2}(P)$ проста.

\section{СПИСОК ЦИТИРОВАННОЙ ЛИТЕРАТУРЫ}

[1] Фукс Л. Частично упорядоченные алгебраические системы. М.: Мир, 1965.

[2] Копытов В. М. Решеточно упорядоченные группы. М.: Наука, 1984.

[3] Higman G. The units of groups rings // Proc. London Math. Soc. 1940. V. 46. № 2. P. 231-248.

[4] Тарарин В. М. О-2-транзитивные группы автоморфизмов с абелевым стабилизатором точки // Матем. заметки. 1999. Т. 65. № 2. С. 289-293.

[5] Фукс Л. Бесконечные абелевы группы. Т. 2. М.: Мир, 1977.

[6] Кокорин А. И., Копытов В. М. Линейно упорядоченные группы. М.: Наука, 1972.

[7] Каргаполов М.И., Мерзляков Ю. И. Основы теории групп. 3-е изд. М.: Наука, 1982.

Институт прикладных математических исследований КарНЦ РАН,

Поступило

Республика Карелия, г. Петрозаводск

16.03.2001

E-mail : tararin@krc.karelia.ru 